Jbrahim Moaned Hamed Abd Ella

Dozent an der Abtcilung für Germanistik

Al-Alsiun Fakultảı

Al-Minia Universitial

Interuationale Tagung der Al-Alsun Fakultä

der Al-Minia Universităt

mit dem Thema: „Das Ägyptenbild in der Weltliteratur“

14 - 15 April 2004

\title{
Zum Bild der altägyptischen Kultur im Leben und Werk des großen deutschen Dichters \\ Goethe
}

In Goethes Gesamtwerk spielt die altägyptische Kultur eine zentrale Rolle. Man hat öfters schmerzlich empfunden, dass die Rezeption der altägyptischen Kultur nicht nachhaltig von Goethes Geist aufgenommen und reflektiert worden ist. Goethe legte großes Gewicht auf die Kultur der Antike. Man meint, Goethe wäre der altägyptischen Kultur gerecht gewesen, wenn er dazu die Möglichkeit erlebt hätte.

Diese Untersuchung befasst sich mit Goethes Verhältnis zur altägyptischen Kultur. Der deutsche Maler Wilhelm Tischbein, der französische Maler Cassas und andern öffneten Goethes Augen auf die alte Kunst Ägyptens. Die Anfänge Goethes Begegnung mit der altägyptischen Kultur erfuhr er von seinen Reisen in Italien. Sein Ägyptenbild erhielt er ferner von seiner eigenen Lektüre, vor alleni, der Lektüre des Winkelmannes Werk "Geschichte der Kunst des Altertums“ sowie des Werkes seines Lehrers Johann Gottfried Herder: „Ideen zur Geschichte der Menschheit". Die beiden Dichter übten auf ihn einen großen Einfluss aus.

\section{I}

"das älteste und herrlichste vieler Monumente"

Am 3. September gleichfalls aus Rom schrieb er über diesen großen ägyptischen Monumenten:

"Ich bin wieder in die ägyptischen Sachen gekommen. Diese Tage war ich einige Mal bei dem großen Obelisken, der noch zerbrochen zwischen Schutt und Kot in einem Hofe liegt. Er war der Obelisk des Sesostris in 
Rom zu Ehren des Augustus aufgerichtet, und stand als Zeiger der großen Somnenuhr, die auf dem Campus Martius gezeichnet war. Dieses ïlteste und herrlichste vieler Monumente liegt nun zerbrochen, einige Seiten wahrscheinlich durchs Feuer verunstaltet. Und doch liegt er noch da, und die unzerstörten Seiten sind noch frisch, wie gestern gemacht und von der schönsten Arbeit (in ihrer Art). Ich lasse jetzt die cine Sphinx der Spitze und die Gesichter von Menschen, Sphinxen und Vögelı abformen und in Gyps gießen.“"

Das zeigt bereits sein reges Interesse an der ägyptischen Kunst. Er studierte das Ägyptische nicht bloß, wo es ihm in Rom vor Augen tritt Eine freudigste Überraschung empfindet man bei der Lektüre der Italienischen Reise (1786/87), in den man einen Gegensatz zu den abweisenden Bemerkungen findet. Hier erlebt man in diesen Sätzen, die von dem ernsten Bemühen Goethes um das Verständnis der altägyptischen Denkmäler, die ihm in Rom begegnete, künden. So war es von seiner Bewunderung und intuitiven Erfassung ihres Sinns.

Durch die Autorität seines Geistes stark reaktiviert als Bildungsmacht vor Augen stellte hat er In Italien vor den ägyptischen Originalen gestanden. Am 29. Oktober 1786 war Goethe durch die Porta del Popolo gefahren und in die ewigen Stadt angekommen. Seine Reisekutsche hatte gewiss auf der berühmten Piazza del Popolo, dem Sammelplatz her Ankommenden, haltgemacht. Und Goethe dürfte hier aussteigen sein, weil sein Quartier bei Wilhelm Tischbein, der am Corso lag, nicht mehr als 300 Schritt entfernt. In Rom wird sein Blick sogleich auf dem Obelisken gefallen sein, der die Mitte des Platzes Campus Martius liegt, ${ }^{2}$ um ihn zu verzieren. Diesen Obelisk hatte einst der römische Kaiser Augustus aus Ägypten nach Rom bringen und im Zirkus Maximus aufstellen lassen. Aus den Trümmern zog ihn dann Papst Sixtus V. hervor und errichtete ihn 1589 aufs neue auf der Piazza del Popolo. Die ägyptischen Monumente, die ihm auf seinen Wegen in Rom, auf Plätzen und in Sammlungen begegneten, suchte er in ihrer Eigenart zu begreifen und zu verstehen. Goethe nannte diesen Obelisk „das älteste und herrlichste vieler Monumente", und ließ sich verschiedene Skulpturen davon in Gips abgießen, denn diese unschätzbaren Sachen müsse man besitzen. In dem späteren Bericht sagt er, dass er:

„[...] überall mit zierlichen naiven Figuren, obgleich in dem bekannten Stil, übersätet sei und macht die überaus feinsinnige Bemerkung, dass die an der Spitze angebrachten Figuren ursprünglich keinem menschlichen

\footnotetext{
' Max Pieper, Ein Goethes Brief über Ägypten. In: Mitteilungen des dt. Institut für Altertumskunde in Kairo, Berlin 1932, S. 127-134. Hier, S. 133.

${ }^{2}$ Brief aus Rom vorn 1. September 1787.
} 
Auge, sondern nur den Strahlen der Sonne erreichbar waren: Hier tritt der Fall ein, dass das Gottesdienstliche der Kunst nicht auf einen Effekt berechnet ist, den es auf den menschlichen Anblick machen soll, $[\ldots]^{\text {‘3 }}$

Jenes von höchster „Vorstelligkeit“ um die von Heinrich Schäfer (Goethes Freund) geprägte Bezeichnung in etwas abgewandeltem Sinne zu gebrauchen zeugende Prinzip, das gewiss auch Goethe tief ergriffen haben würde, wenn er es in ägyptischen Gräbern zu äußerster Konsequenz durchgeführt hätte sehen können. Und das „wahrnehmige“ Menschen zunächst so fremdartig berührt, wenn wir etwa von dem Grundsatz Friedrich Theodor Vischers herkommen, dass "Schönheit ein Akt ist“, insofern sie im Auge des betrachtenden Menschen erst jeweilig entsteht.

II

Mit seinem Hausgenossen Wilhelm Tischbein, dem Maler, hat Goethe in jenen Tagen um Jahreswende 1786/87 wahrscheinlich des öfteren über seine Beobachtungen an ägyptischen Dingen gesprochen. Es war eine seltene und teure Überraschung, dass Goethes Freund, der begabte Maler Wilhelm Tischbein auf den Gedanken gekommen ist. Dass iln sein Freund, Goethe auf einem Obelisken sitzend zu malen. Goethe notiert am 29. Dezember 1786 über diesen Plan:

"Ich bemerkte wohl, daß Tischbein mich öfters aufinerksam betrachtete, und nun zeigt sich's, daß er mein Porträt zu malen gedenkt. Sein Entwurf ist fertig; er hat die Leitwand schon aufgespannt. Ich soll in Lebensgröße, als Reisender, in einen weißen Mantel gehülit, in freier Luft, auf einem umgestürzten Obelisken sitzend, vorgestellt werden, die tief im Hintergrunde liegenden Ruinen der Campagna die Roma überschauend. Es giebt ein schönes Bild, nur zu groß für unsere nordischen Wohnungen. Ich werde wohl wieder dort unterkriechen; das Portrait aber wird keinen Platz finden. ${ }^{* 4}$

Diese von Goethe selbst niedergeschriebene Entstehungsgeschichte des berühmtesten Gemäldes von Wilhelm Tischbein und eines der bekanntesten Goethebildnisse ist offenbar deswegen bisher wenig beachtet worden, weil die endgültige Ausführung des Bildes den umgestürzten Obelisken als solchen nicht mehr erkennen lässt, sondern Goethe auf zwei einfachen Blöcken sitzend darstellt. Die ersten Entwürfe aber zeigten einen in zwei Teile zerbrochenen, liegenden

\footnotetext{
${ }^{3}$ Ludwig Volkmann: Goethe und Ägypten. In: Zeitschrift für ägyptische Sprache und Altertumskunde, Leipzig 1936, von Seite 1 - 20. Hier S. S. 8.

${ }^{4}$ Zit. nach Karl Heinrici Dittmann. Goethe und die „Egyptischen Sachen“. In: Mittelungen des deutschen Instituts für Ägyptische Altertumskunde. Kairo 1943, S. 96-106. Hier S. 97f.
} 
Obeliskenschaft, über und über mit Hieroglyphen bedeckt. Im Goethehaus in Weimar wird einer dieser Erntwürfe, eine Skizze aufbewahrt.

Goethe selbst hat im Jahre 1821 ein Verzeichnis der Tischbein-Skizze angefertigt, der in seinem Besitz gefunden wurde. Und beschreibt darin das Blatt mit den Worten: „[...] der Reisende selbst im weißen Mantel, auf zerbrochnem Obelisk sitzend, die Campagna die Roma dahinter [...]. “5 Auf die ursprüngliche Fassung des Bildes geht eine noch wälırend Goethes Aufenthalt in Rom von F. Bury. H. Meyer und J. G. Schütz gemeinsam angefertigte Aquarellkopie im Weimarer Goethehaus zurück, die ihrerseits wieder Vorbild für eine von J. Siebert entworfene und durch j. Brodtmann ausgeführte Lithographie wurde. ${ }^{6}$ Von der Lithographie sind Abzüge in mansche Sammlung gelangt. Die Lithographie und ihr Aquarellvorbild überliefern also Tischbeins erste Komposition, wie Goethe sie in der Italienischen Reise und in Verzeichnis seiner TischbeinSkizzen beschreibt und wie sie in zeitgenössischen Briefen dargestellt wird. $^{7}$

$\mathrm{Zu}$ der Idee, Goethe auf einem liegenden Obelisken sitzend zu malen, konnte Tischbein im damaligen Rom sehr wohl angeregt werden. Denn noch waren nicht alle während der römischen Kaiserzeit aus Ägypten nach Rom verschleppten und später wieder gestürzten Obelisken aus dem Schutt der antiken Trümmer gezogen und aufgerichtet worden. Wie oft mag Tischbein Goethe auf dem zerbrochen liegenden Obelisken auf dem Marsfelde sitzend gesehen und beobachtet haben.

Der Weg der beiden Freunde führte öfter dorthin, und einige Monate nach dem Beginn des Gemäldes, während Goethes zweiten Aufenthaltes in Rom, ergab sich wiederum ein besonders Anlass, den gefallenen Obelisken, den Augustus einst auf dem Campus Martius hatte errichten lassen, aufzusuchen.

III

„Es ist diese Zeichnung die ungeheuerste Architecturidee, die ich zeitlebens gesehen, und ich glaube nicht, dass man weiter kann."

Der Französische Architekt Louis Francois Cassas ${ }^{8}$ hatte im Sommer 1787 eine Studienreise durch Türkei, Syrien, Palästina und Ägypten beendet und hielt sich auf dem Rückwege in sein Vaterland Frankreich einige Zeit in Rom auf. Er brachte eine Mappe mit Zeichnungen und Farbskizzen von den besuchten Landschaften, Ruinen und Städten und

\footnotetext{
${ }^{5}$ Ebd. S. 98.

${ }^{6} \mathrm{Vgl}$. Den Katalog des Staedelschen Kunstinstituts in Frankfurt am Main von Jahre 1900.

${ }^{7}$ Vgl. Karl Heinrich Dittmanu. Goethe und die „Egyptischen Sachen“. In: Mittelungen des deutschen Instituts für Ägyptische Altertumskunde. Kairo 1943, S. 96-106. Hier S. 98.

${ }^{8}$ Geboren im Jahre 1756 und gestorben im Jahre 1787.
} 
hatte es als Architekt und Bauforscher nicht unterlassen, sich gelegentlich von den zerstörten Stätten des Altertums den ursprünglichen Zustand vorzustellen und ihn nach seiner Phantasie aufs Papier zu bringen. Goethe wurde mit ihm bekannt und ließ sich seine Entwürfe zeigen. Am 3. September 1787 berichtet Goethe davon:

Ein französischer Architekt, mit Namen Cassas, kam von seiner Reise in den Orient zurück; er hatte die wichtigsten alten Monumente, besonders die noch nicht herausgegebenen, gemessen, auch die Gegenden, wie sie anzuschauen sind, gezeichnet, nicht weniger alte zerfallene und zerstörte Zustände bildlich wieder hergestellt und einen Theil seiner Zeichnung, von großer Präcision und Geschmack, mit der Feder umrissen und mit Aquarellfarben belebt dem Auge dargestellt. ${ }^{9}$

Es folgt dann eine Aufzählung und Beschreibung von zehn Zeichnungen, die Cassas ihm vorgelegt hat. Man nennt hier nur die einzelnen Gegenstände und geben erst bei 9 und 10 wieder Goethes vollständigen Text:

1. Das Serail von Constantinopel...

2. Generalaussicht der Ruinen von Palmyra...

3. Gräber aus Palmyra...

4. Restauration des Sonnentempels zu Balbeck...

5. Die große Mosche zu Jerusalem...

6. Ruinen eines kleinen Tempels in Phönicien...

7. Gegend am Fuße des Berges Libanon...

8. Türkische Gräber...

9. Ägyptische Pyramide mit dem großen Sphinxkopfe. Er sey, sagt Cassas, in einem Kalkfelsen gehauen, und weil derselbe Sprünge gehabt und Ungleichheiten, habe man den Koloß mit Stuck überzogen und gemalt, wie man noch in den Falten des Kopfschmuckes bemerke. Eine Gesichtspartie ist etwas 10 Schuh hoch. Auf der Unterlippe hat er bequem spazieren können.

10.Eine Pyramide, nach einigen Urkunde, Anlässen und Muthmaßungen restauriert. Sie hat von 4 Seiten vorspringende hallen mit danebenstehenden Obelisken; nach den Hallen gehen Gänge hin, mit Sphinxen besetzt, wie sich solche noch in Oberägypten befinden. Es ist diese Zeichnung

\footnotetext{
${ }^{9}$ Max Pieper. Goethes Urteilen über ägyptische Kunst und Religion. In: Mitteilungen des Deutschen Instituts für Ägyptische Altertumskunde II., Kairo 1932, S. 117- 134.
} 
die ungeheuerste Architecturidee, die ich zeitlebens gesehen, und ich glaube nicht, daß man weiter kann. ${ }^{10}$

Dazu hatte Cassas eine Pyramidenanlage rekonstruiert. „Sie hat von vier Seiten vorspringende Hallen mit danebenstehenden Obelisken, nach den Hallen gehen Gänge, mit Sphinxen besetzt, wie sich solche noch in Oberägypten befinden." Goethe fügt hinzu: „Es ist diese Zeichnung die ungeheurste Architekturidee, die ich zeitlebens gesehen, und ich glaube nicht, daß man weiter kann. "ll Das ist eine bewundernde Anerkemnung altägyptischer Architektur. Goethe zeigt hier, wie man sagen kann, ein Wirkliches Verständnis ihrer Größe, dem dadurch nichts genommen wird, class hier Richtiges und Falsches wunderlich durcheinandergemischt ist. Richtig ist das Gefühl, dass eine Pyramide allein undenkbar ist, dass da noch bauten zurückgehören, wit man seit einigen Jahrzehnten ja positiv wissen. Es wäre interessant eine der Frage nachzugehen, woher Cassas auf diesen Gedanken gekommen sind. Ein verschollenes Reisewerk ,von Le Masquier" hatte noch Spuren von dem Aufgang zu den Pyramiden erwähnt, man mïssste zu erfahren suchen, ob diese Beobachtung weiter gegeben ist. Unmittelbar hinter den erwähnten Ausführungen Goethes findet sich eine merkwürdige Stelle über den vorher erwähnten Obelisken:

„Er ist aus echtestem ägyptischen Granit gehauen, überall mit zierlichen naiven Figuren, obgleich in dem bekannten Stil übersäet. Merkwürdig war, als wir neben der sonst in die Luft gerichteten Spitze standen, auf den Zuschärfungen derselben Sphinx nach Sphinx a:if das zierlichste abgebildet zu sehen, früher keinem menschlichen Auge als den Strahlen der Sonne erreichbar. Hier tritt der Fall ein, daß das Gottesdienstliche der Kunst nicht auf einen Effekt berechnet ist, den es dem menschlichen Auge machen sollen. “12

Goethe erzählt dann weiter, wie er in der großen Dehnischen Pastensammlung sich Abdrücke ägyptischer Gemmen erworben und bestellt habe. Also doch wohl die Abraxasgemmen, über die er später ein so ungünstiges Urteil fällte. Doch muss auch mit der Möglichkeit gerechnet werden, dass es sich um ägyptische Skarabäen handelte. Cassas versieht seine Phantasie-Pyramide zusätzlich noch mit Säulenhalle, Obelisken und einer Sphinx-Allee. Die Rekonstruktion der

\footnotetext{
${ }^{10}$ Ebd. S. 133.

1 Zit. nach Ludwig Volkmann: Goethe und Ägypten. In: Zeitschrift für ägyptische Sprache und Altertumskunde, Leipzig 1936, von Seite 1 - 20. Hier: S. 9.

${ }_{12}$ Max Pieper. Goethes Urteilen über ägyptische Kunst und Religion. In: Mitteilungen des Deutschen Instituts für Ägyptische Alt_rtunskunde II., Kairo 1932, S. 117- 134. Hier: S. 133.
} 
Pyramidenanlage - wohlgemerkt, nicht auf archäologische Beobachtungen oder Funde gegründet ${ }^{13}$ - hat in ihrer phantastischen Konzeption die größte Wirkung auf Goethe ausgeübt. Sie wirklich eine „ungeheure Architekturidee" wieder und konnte, wenn auch nicht als wahr, so doch als gut erfunden gelten, um den Betrachtern jener Zeit einen Eindruck von der Gewalfigkeit altägyptischer Bauten zu vermitteln. Cassas hatte in dieser Zeichnung sein eigenes Erleben vor den Pyramiden und Tempelresten in einem großartigen Entwurf zusammengefasst. Da Goethe an diesem Erlebnis teilhatte, lohnt es sich, nach dem Verbleib der Cassasischen Zeichnung zu suchen. Sie ist als Kupferstich in sein großes Reisewerk aufgenommen worden, das er nach seiner Rückkehr in Paris zusammenstellte und unter dem Titel „Voyage Pittoresque de la Syrie, de la Phénicie, de la Palastine et de la Basse - Egypte" 1799 veröffentlichte. Auf einem Bild als zentraler Hauptbau erhebt sich die große Pyramide im Hintergrunde, an ihrem Fuß sehen wir die vorspringende Halle mit danebenstehenden Obelisken. Auf sie führt eine breite Prozessionstrasse, die links und rechts von Sphinxallee gesäumt wird und Schauplatz einer sich gerade abspielenden großen heiligen Handlungen ist.

Es darf einmal Goethes Schawen und Erkennen an einem Einzelbeispiel vom ersten Anlass aus über Begeisterung bis zum reifen Gedanken hin miterleben. Der Weg führt von der Betrachtung ägyptischer Denkmäler über die Lektüre Winkelmanns und die Begegnung mit ägyptischer Architekturidee zum wesentlichen Begreifen ägyptischer Kunst und zum Wunsche, auch weiterhin ihre Werke vor Augen zu sehen. An das Interesse für die Zeichnungen des französischen Architekten ist so kurz von Goethe erinnert. Aber mit dem wichtigen Urteil über die Rekonstruktion einer Pyramiden-Anlage mit Obelisken, Hallen, und Sphinx-Allen: „Es ist diese Zeichnung die ungeheuerste Architekturidee, die ich zeitlebens gesehen, und ich glaube nicht, daß man weiter kann. "'14 Die Vollständigkeit kann noch halber angeführt sein, dass in den Fragmenten über Italien zum „Material der bildenden Kunst“ besonders auf die ägyptischen Obelisken hingewiesen wird, deren Form durch die natürliche Struktur des Granits mitbedingt ist. ${ }^{15}$ So über die Obelisken konnte Goethe bei Volkmann so manche Angaben finden.

Die Niederschrift von Goethe über Cassas und den Obelisken auf dem Marsfelde ist einer der sogenannten Berichte, die in die für den Druck bestimmte Redaktion der Italienischen Reise 1813 von ibm eingeschoben worden, auf Briefen, Reisenotizen und Tagebucheintragungen beruhen.

\footnotetext{
${ }^{13}$ Max Pieper hat mit Recht anerkannt, dass Cassas die Pyramiden als zentrale Tcile größerer Bauzusammenhänge ansah, was für damalige Zeit, die keinem archäologischen Anlass zu diesem Gedanken kannte, eine überraschend richtige Idee darstellt.

${ }^{14} \mathrm{Zit}$. nach Ludwig Volkmann: Goethe und Ägypten. In: Zeitschrift für ägyptische Sprache und Altertumskunde, Leipzig 1936, von Seite 1-20. Hier: S. 9.

${ }^{15}$ Ebd. S. 9
} 
Er zeigt, dass das Erlebnis noch 1813 Goethe lebendig vor Augen stand. Es scheint, dass Goethe die schöne Erkenntnis über die auch in der Verborgenheit sich vollziehende ,gottesdienstliche“ Wirkung ägyptischer Kunstwerke erst 1813 zugewachsen ist, da der mit dem Bericht gleichzeitig datierte, weiter im Rom-Brief, der den gleichen Besuch beim Obelisken behandelt. Ein auch 3. September 1787 aus Rom datierter Brief, der gleichfalls im zweiten Teil der Italienischen Reise Aufnahme gefunden hat, bestätigte den Bericht und legt noch einmal Zeugnis ab von Goethes Ergriffenheit:

Diese unschätzbaren Sachen muß man besitzen, besonders da man sagt, der Pabst wolle ihn aufrichten lassen, da man denn die Hieroglyphen nicht mehr erreichen kann. So will es auch mit den besten Etrurischen Sachen thun u. s. w. Nun modelliere ich nach diesen Bildungen in Thon, um mir alles recht eigen zu machen. ${ }^{16}$

In diesen Zeilen verbindet sich Goethes Rom-Erlebnis in seiner Gesamtheit mit den Zeugen ägyptischer Kunst. Er zieht die „Egyptischen Sachen" hinein in sein Begreifen der klassischen Welt der ewigen Stadt. Ägyptische Werke werden ein Teil seiner römischen Bildung. Er machte sie sich „zu eigen“ in seiner besonderen Art, verschafft sich Abgüsse der Reliefbilder des Obelisken, der Sphinxdarstellungen an seiner Spitze und der Hieroglyphen an seinen Steinflächen, um diese „unschätzbaren Sachen zu besitzen", ebenso zu besitzen wie den kolossalen Jupiterkopf oder die Medusenmaske oder die „Etrurischen Sachen“. Mehr noch modelliert er die Figuren mit eigener Hand in Ton, um sich ihrer geistig zu vergewissern. An Charlotte von Stein schreibt er am 19. Januar 1788:

Wenn du nur einen Abend bey uns seyn solltest unter den vielen Gipssachen, wenn man die besten Sachen neben einander setzen kann und sich das für treffliche vom Guten so sehr, ja unendlich absondert. Ich spreche nicht aus, wie glücklich ich bin da zu sehen anfange, wo ich Zeitlebens nur getappt hab. ${ }^{17}$

\section{IV}

Es soll zu erwähnen, das der Obelisk als ein ägyptisches Monument also war, das Goethe als erste Merkwürdigkeit bei seinem Einzug in Rom wahrnehmen konnte. Wenige Wochen nach seiner Ankunft am 4. Januar 1787 schreibt er:

\footnotetext{
${ }^{16}$ Max Pieper. Goethes Urteilen über ägyptische Kunst und Religion. In: Mitteilungen des Deutschen Instituts für Ägyptische Altertumskunde II., Kairo 1932, S. 117- 134. Hier: S. 133

${ }^{17}$ Goethes Briefe. Bd. 2, Brief vom 19. Januar an Charlotte von Stein. Brief Nr. 1639, S. 259.
} 
Winckelmanns Geschichte der Kunst habe ich angefangen zu lesen, und habe erst Egypten zurückgelegt, und fühle wohl, daß ich nun erst wieder von vorn sehen muß; auch habe ich in Absicht auf die Egyptische Sachen gethan. Je weiter herauf, desto unübersehlicher wird die Kunst, und wer sichere Schritte thun will, muß sie langsam thun. ${ }^{18}$

Goethe war zugleich der Größte überhaupt, der sich an Winckelmann und Herder gebildet hat. Es ist seltsamen Reiz zu beobachten, dass die Wirkung der zwei großen auf Goethe gerade in besonderer Weise spürbar wird. Um es vorwegzunehmen: Goethes Verhältnis zu Ägypten hat sich augenscheinlich gewandelt, in dem seine inneren Voraussetzungen zunächst von dem einen, dann von dem anderen bestimmt worden sind. Im Banne der von Winckelmann rezipierten antiken Vierheit, die Ägypten in ihrem System barg.

„Ich hab angefangen, Winckelmanns Kunstgeschichte zu lesen und fühle wohl, dass ich nun erst wieder von vorn selsen muß, auch hab ich in Absicht auf die ägyptischen Sachen getan. "19 So schreibt er aus Rom im Januar 1787.

Hier soll man erwähnen, dass Goethe zu dieser Lektüre von Winckelmanns Kapitel „Von der Kunst unter Ägyptern“" zweifellos durch Johann Volkmanns „Nachrichten von Italien“ (1770) bestimmt worden war, denn er benutzte dieses damals grundlegende Werk ja dauernd und bezieht häufig darauf; ${ }^{20}$ hatte es dort in Bezug auf die ägyptischen Figuren im Bodengeschoss der kapitolinischen Sammlung lesen können. Also Volkmanns Buch ist überhaupt sehr Aufschlussreich über das, was Goethe in Rom an ägyptischer Kunst sehen bzw. nicht sehen konnte. ${ }^{21}$

Als Goethe von Deutschland abreiste, nahm er die Anschauung der antiken Kunst, wie Winkelmann sie seinem Jahrhundert eröffnet hatte, im Geiste und im Herzen mit. Aber erst in Rom begann Goethe mit dem Vermächtnis Winkelmanns zu leben, mit Winckelmanns Augen die Kunst der Antike in ihrer ganzen Fülle zu sehen. In seinem Gepäck führte er ein Reisehandbuch mit ihm, das in anschaulicher, auch heute noch mit Genuss lesbarer Form alles bemerkenswerte in Italien beschreibt und erläutert und zu seiner Zeit großen Ruhm hatte: Johann Jacob Volkmann, Historisch-Kritische Nachrichten von Italien, welche eine Beschreibung dieses Landes, der Sitten, der Regierungsform, Handlung, des Zustandes

${ }^{18}$ Goethes Briefe. Bd. 2, Brief an Charlotte von Stein. Brief Nr. 1605. S. 201.

${ }^{19}$ Max Pieper. Goethes Urteilen über ägyptische Kunst und Religion. In: Mitteilungen des Deutschen Instituts für Ägyptische Altertumskunde II., Kairo 1932, S. 117- 134. Hier: S. 132.

${ }^{20}$ Im II. Band, S. 491.

${ }^{21}$ Vgl. Ludwig Volkmann: Goethe und Ägypten. In: Zeitschrift für ägyptische Sprache und Altertumskundc, Leipzig 1936, von Seite 1-20. Hier S. 8. 
der Wissenschaften und insbesondere der Werke der Kunst enthalten. In diesen nützlichen Werke steht im zweiten Bande. Ein Absatz über die ägyptische Sammlung, die Benedikt XIV. im Bodengeschosse des Kapitols angelegt hatte, und in einer Fußnote dazu ist zu lesen:

„Um diese Sammlung mit rechtem Nutzen zu besehen, muß man das andere Kapitel in Winckelmanns Geschichte der Kunst, welches vom ägyptischen Stil handelt, lesen. Man wird die Sachen alsdann mit viel aufgeklärten Augen ansehen" ${ }^{22}$

Dieser Rat ist, wie wir sehen, von Goethe befolgt worden. Seine eigene Worte, dass er ,nun erst wieder von vorn sehen ${ }^{\text {‘23 }}$ müsse, dürften der Widerhall von Volkmanns Anmerkung sein. Sie lasen zugleich erkennen, dass Goethe die neue Welt der ägyptischen Kunst, die Winckelmanns "Geschichte der Kunst des Altertums" mit dem ersten Abschnitt des zweiten Kapitels „Von der Kunst unter den Ägyptern" ihm zugänglich machte, auf sishı wirken ließ. Und dass sie ihn veranlasste, seine Kunstanschauung zu revidieren. ${ }^{24}$ „Auch habe ich es in Absicht auf die Egyptischen Sachen gethan".

Die Besuche Goethes auf dem Marsfelde verbindet ihm mehr und mehr mit Winckelmann, dessen Nachwirkung auf Goethe in diesem Fall wieder spürbar wird. Winckelmann hatte doch auch einige Jahrzelnnte vorher den Obelisken gesehen und die Kunst seiner Figuren gerühmt. Im Abschnitt über die ägyptische Kunst konnte Goethe nach einer Erwähnung der Hieroglyphen an der Basis eines Granitsphinx in Dresden die Worte lesen: „Die Sphinxe an dem Obelisken der Sonnen, welcher in Campo Marzo liegt, sind in eben dem Stil, und in den Köpfen ist eine große Kunst und Fleiß“, und über dem Anfang des Kapitels sah er den Kupferstich eines dieser Sphinxe, von denen er dann einen in Gips abformen ließ. Zu der Sphinxabbildung in seiner Kunstgeschichte hatte Winkelmann die Anmerkung geschrieben:

„Zu Anfang des zweiten Kapitels steht der Sphinx an der Spitze des Obelisken der Sonne, welchen Augustus nach Rom bringen ließ. Es liegt derselbe zerbrochen und vom Feuer sehr beschädigt an dem Orte, wo er gefunden worden. Dieser Sphinx ist hier als eines der ältesten Werke der ägyptischen Kunst angebracht. [...] $]^{\text {‘25 }}$

\footnotetext{
${ }^{22}$ Zit. nach Karl Heinrich Dittmann. Goethe und die „Egyptischen Sachen“. In: Mittelungen des deutschen Instituts für Ägyptische Altertumskunde. Kairo 1943, S. 96-106. Hier: S. 97

${ }^{23}$ Goethes Briefe. Bd. 2, Brief an Charlotte von Stein, Brief Nr. 1605. S. 201

${ }^{24} \mathrm{Vgl}$. Vgl. Karl Heinrich Dittmann. Goethe und die "Egyptischen Sachen“. I deutschen Instituts für Ägyptische Altertunskunde. Kgyptisch

${ }^{25}$ Zit. nach Karl Heinrich Dittmann, S. 97.
} 
Der Einfluss auf Goethe, ja sogar auf seine Worte, ist unverkennbar. Der Verbundenheit mit dem Werke Winckelmanns, der die Antike für den Deutschen aufs neue heraufbeschworen und ihr eine neue Gültigkeit für das deutsche Geistesleben verliehen hatte, setzte Goethe 1805 sein wundervolles Denkmal, das mit den Worten abschließt: „Von seinem Grabe her stärkt uns der Anhauch seiner Kraft und in erregt in uns den Lebhaftesten Drang, das, was er begonnen, mit Eifer und Liebe fort - und immer wieder fortzusetzen -,

Goethe hat, wie wenige, im Geiste Winckelmanns gedacht und sein Denken fortgeführt. So verwundert es nicht, dass auch die Erinnerung an die in Rom geschaute ägyptische Kunst noch einmal aufklingt. Und das gerade in jener Schrift über Winckelmann im zweiten Aufsatz, wo Goethe die von Winckelmann im Anschluss an die Arbeiten des Grafen Caylus $^{26}$ gesetzte Periodeneinteilung der ägyptischen Kunst anführt: „Noch werden die Werke des ägyptischen Geschmackes in drei Classen, nämlich in echt ägyptische, und ferner in griechische und römische Nachahmungen des ägyptischen Geschmackes eingetheilt; die Kennzeichen aber der früheren und späteren Werke jener ersten Classe sind noch immer nicht erforscht" ${ }^{27}$ Winckelmanns Periodeneinteilung lautet allerdings etwas anders, als Goethe sie formuliert:

„In den zwei ersten (der drei Absätze) wird gehandelt vom dem älteren und nachher vom dem folgenden und späteren Stil der ägyptischen Bildhauer und in dem dritten Absatze von den Nachahmungen ägyptischer Werke, durch griechischen Künstler gemacht. Ich werde ... darzutun suchen, daß die wahren alten ägyptischen Werke von zweifacher Art sind, und daß man in ihrer eigenen Kunst zwei verschiedene Zeiten setzen müsse: die erste hat vermutlich gedauert, bis Ägypten durch den Cambyses erobert wurde, und die zweite Zeit, solange eingeborne Ägypter unter der persischen und nachher unter der griechischen Regierung in der Bildhauerei arbeiteten; die Nachlahmungen aber der ägyptischen Werke sind vermutlich alle unter dem Kaiser Hadrian gemacht. ${ }^{c 28}$

Diese Erkenntnis bedeutet wirklich Fortsetzung Winckelmanns Denken und ist es nichts Geringeres als die Aufgabenstellung der ägyptischen Kunstforschung des 19. und 20. Jahrhundert. Es war vielmehr schon hervorgehoben worden, dass Ägypten in dessen „Geschichte der Kunst

${ }^{26}$ Er hatte ein Buch im Jahre 1752 - 1768 in Paris unter dem Titel „Recueil de Antiquites Egyptiennes, Etrusques, Grecques et Romaines verfasst.

${ }^{27}$ Zit. nach Karl Heinrich Dittmann. Goethe und die „Egyptischen Sachen“. In: Mittelungen des

deutschen Instituts für Ägyptische Altertumskunde. Kairo 1943, S. 96-106. Hier: S. 102.

${ }_{28}^{28}$ Aus dem Winckelmanns Buch „Geschichte der Kunst des Altertums“ (1764). 
des Altertums“ (1764) seinen Platz gehabt. Dass Winkelmann bei alledem noch in der Tradition von der Ägypten einschließenden Vierheit der Alten Welt gestanden habe. Freilich ist es eben diese Vierheit, genauer: die überragende Stellung von Hellas in ihrem System gewesen, die Ägypten nicht recht zur Geltung kommen ließ. Was Winckelmann für die Methode einer stilgeschichtlichen, das Lebensganze berücksichtigenden Kunstforschung geleistet hat, macht vor den ägyptischen Dingen nicht halt. Man sieht Winckelmanns Begegnungen mit ägyptischen Originalen in Rom, er benutzte vorliegende Denkmäler, Publikationen und Reiseberichte kritisch und zieht antike Autoren seit Herodot heran. Seine Untersuchungen versclımälıen die äußeren Dinge nicht Material, Werkverfahren, Tracht und denken an andere Lebensbereiche (Religion). Er mülht sich um Deutung der einzelnen Werke und strebt ilire historische Ordnung an, d. h. er erkennt, dass auch die ägyptische Kunst ein historisches Phänomen sein muss. ${ }^{29}$ Ägypten ist ihm kaum mehr als eine Folie für Hellas. Größe und Grenze seiner Einbeziehung Ägyptens bestehen darir, dass die stilgeschichtliche Ordnung von Kunstwerken, die er als solche geschaffen hat, Ägypten im Prinzip zugute kam, dass aber die echte Maßstäblichkeit unmöglich wurde. Das wird dann auch im Hinblick auf die Hieroglyphen deutlich. Winckelmann begnügt sich noch kurz vor seinem Tode mit dem Gemeinplatz des 18. Jahrhunderts, dass man die ägyptische Schrift nach dem Scheitern Kirchers nicht enträtseln könne. $^{30}$

Winckelmanns Buch, das über dem Anfang des ersten Kapitels „Von dem Ursprung der Kunst" steht und besonders bezeichnend für die Zusammenschau der Antike zu sein scheint. Es zeigt eine halb im Boden steckende griechische Säule, einen bärtigen römischen Sphinx, einen ,ertrurischen Krater und eine umgefallene ägyptische Statue, dazu ein Relief und ein paar Münzen oder „geschnittene Steine“. Als Symbole für die Künste, über die der Leser in einer Geschichte der Kunst des "Alterthums“, die 1767 erschien, erwarten konnte, belehrte zu werden. Ihre Zusammenstellung auf einem Bild illustriert den einheitlichen Begriff „Altertum“ und zeigt, welche Kunstgruppen das 18. Jahrhundert darunter verstand. Dieselbe Einheit will die Titelvignette der zweiten Ausgabe von Winckelmanns Kunstgeschichte (1776) ausdrücken. Sie fasst die römische Wölfin, einen „etrurischen“ Topf, die Büste Homers und einen ägyptischen Sphinx zusammen. Ein Ausblick auf die Kunst der Phönizier und Perser, wie Winckelmann ihn gibt, ist innerhalb dieser

\footnotetext{
${ }^{29}$ Siegfried Morenz. Dic Begegnung Europas mit Ägypten. Züriclı und Stuttgart 1969, S. 136.
}

${ }^{30}$ Ebd. S. 137. 
Vierheit möglich. Er erweitert aber nicht das einmal vor Augen stehende Bild der Antike, zumal er kurz abgetan wird. ${ }^{31}$

Wie Winckelmann, so erlebt auch Goethe die ägyptische Kunst in Rom als Glied der klassischen Antike. Denn dies ist das geistesgeschichtliche Entscheidende: der Begriffsinhalt der Antike, wie er für Winckelmann und sein Jahrhundert maßgebend war, umfasst Ägypten, Griechenland, Etrurien und Rom. Bei allem Wissen um die Unterschiede dieser Welten, bei allem Vorzug, der Rom und insbesondere Griechenland zuteil wird, für die gelehrte Arbeit sowohl wie für das Geschichtsbewusstsein und das künstlerische Denken verbanden die vier sich zu der Einheit des Altertums schlechthin. Und dieser so gefasste Begriff des Altertums wuchs jener Zeit aus den antiken Schriften und den Ruinen Roms zu. „Rom ist der Ort, in dem sich für unsere Ansicht das ganze Altertum in Eins zusammenzieht", diese Worte eines Freundes, die Goethe in seinem Winckelmann anführt, kennzeichnen seine und seiner Zeitgenossen Auffassung. Mehr noch als die programmatischen Buchtitel, wie der des genannten Recueil de antiquite des Grafen Caylus als Denkmälerpublikation orientierte Werke. Es soll zu erwähnen, dass das Werk von L. Rocchegiani, Costumi religiosi, civili e militari degli antichi Egyziani, Etrusci, Greci e Romani die barocken Vignetten, mit denen Titelblätter gewähren und Kapitalanfänge geschmückt wurden, Einblick in die Denkbahnen des Jahrhunderts. ${ }^{32}$

$\mathrm{V}$

Im Jahre 1784 erschien die Fortsetzung von Herders Universalgeschichte mit dem Ägyptenkapitel, der Goethe noch in Italien mit Spannung entgegengesehen hat. Offensichtlich unter dem Eindruck ihrer Sprengkraft gegenüber der als verbindliche Bildungsmacht überlieferten antiken Vierheit hat sich Goethe später gegen Ägypten verschlossen. Das klingt eirfach und ist gewiss auch die wesentliche Linie. Dann möchte man zunächst die Hauptlinie, also die Abkehr Goethes von Ägypten unter Herders Einfluss, durch einen simplen Gedankenzug unterstützen: Waren die ägyptischen Dinge ins grenzenlose Allgemeine und vorläufig in keiner Weise Bewältigte hinausgestoßen, so konnten sie den Rang einer Bildungsmacht nicht behaupten und jedenfalls für Goethe noch nicht auf einer denkbaren neuen Ebene wiedergewinnen. Daher wurde sie ihm buchstäblich zu Kuriosität:

„Chinesische, Indische, Ägyptische Altertümer sind immer nur Curiositäten; es ist sehr wohlgethan, sich und die Welt damit bekannt zu

\footnotetext{
${ }^{31}$ Vgl. Karl Heinrich Dittmann. Goethe und die „Egyptischen Sachen“. In: Mittelungen des deutschen Instituts für Ägyptische Altertunskunde. Kairo 1943, S. 96-106. Hier: S. 103.

${ }^{32}$ Ebd. S. 103.
} 
machen; zu sittlicher und ästhetischer Bildung aber werden sie uns wenig fruchten. “"33

Schon lange vorher hatte er geschrieben:

„Mit den ägyptischen Altertümern mache man $\underset{\mathbf{3} 34}{\text { sich aus }}$ Reisebeschreibungen mit so wenig Kosten als möglich bekannt. “34

Wenn man dazu Goethes geistige Lebensmaxime in Betracht zieht, die er Schillern gegenüber formuliert hat: dass ihm alles verhasst sei, was nur sein Wissen vermehre, olne ihn wirklich zu bilden, so lässt sich seine Haltung gegenüber dem von Herders Sprengkraft im Prinzip entzauberten Ägypten gut verstehen. Olıne solche Bezüge auf Goethes Lebensmitte würden die einzelnen Äußerungen im leeren Raum schweben.

Von Neapel aus schreibt Goethe am 27. Mai 1787, wenige Tage vor seinem zweiten Aufenthalt in Rom:

„Auf Herders dritten Theil freu sich mich sehr: Hebt mir ihn auf bis ich sagen kann, wo er mir begegnen soll!، ${ }^{35}$

Herders „Ideen zur Geschichte der Menschheit“ begannen 1784 zu erscheinen. Sie stellten die Erde als Planeten in den Weltenraum, den Menschen als Geschöpf unter Geschöpfe, betrachteten Volk um Volk, in dem sie von Kontinent zu Kontinent, von Landstrich zu Landstrich führten und Umwelt, Zeitbedingung und Veranlagung als lebendig wirkende geschichtliche Kräfte aufwiesen. Herders „Ideen“ bezeichnen den Anfang der universalen Geschichtsschreibung, in der die geschichtlichen Erscheinungen der Welt ihre Ordnung und ihren Platz im Gesamtgeschehen erhalten:

„Setzet lebendige Menschenkräfte in bestimmte Verhältnisse ihres Orts und Zeitmaßes auf der Erde; und es ereignen sich alle Veränderungen der Menschengeschichte. Hier krystallisieren sich Reiche und Staaten, dort lösen sie sich auf, und gewinnen andere Gestalten; und das alles in einer Weltgegend, in nachbarlicher Nähe gegen einander. Nur Zeiten, nur Örter und Nationalcharaktere, kurz das ganze Zusammenwirken lebendiger Kräfte in ihrer bestimmten Individualität entscheidet, wie über alle Erzeugungen der Natur, so über alle Ereignisse im Menschenreiche. ${ }^{\text {36 }}$

${ }^{33}$ Wilhelm Meisters Wanderjahre, Buch 3. Zit. nach Morenz. Die Begegnung Europas mit Ägypten.

Stuttgart und Zürich 1969, S. 140., Volkmann, S. 1f.

${ }^{34}$ Brief vom 22. 2. 1797 an Schleusner. Zit. von Volkmann, S. 7.

35 Volkinann, S. $6 \mathrm{f}$.

${ }^{36}$ Ebd. S. 7. 
Herders dritter Teil seiner „Idee“ auf dessen Erscheinen Goethe sich freute, kam 1787 heraus und erhielt Buch XI bis XV. Das XII. Buch enthielt neben Babylon, Assyrien und Chaldäa, neben Meder, Persern und Palästinenser auch ein Abschnitt über Ägypten. Goethe hat das Buch während seiner Rückreise aus Italien gelesen. Ägypten reihte sich hier als Land unter Ländern ein, die Ägypter stellten sich als Volk unter anderen Völkern dar und wurden keineswegs als besondere geschichtliche Erscheinung charakterisiert. Im Gegenteil, ihre cinmaligen Leistungen schrumpfen zusammen, ihre Pyramiden wurden auf ursprüngliche rohe Steinhaufen zurückgeführt, ihre Hieroglyphen als primitive Versuche des menschlichen Verstandes erklärt, ilhren Bildwerken wurde Anmut abgesprochen. Sie rückten in das Kindesalter der Menschheit hinauf, aus dem heraus sie sich niemals entwickelt hätten. Herder schreibt:

"Schwerlich würde Ägypten in den hohen Ruf seiner Weisheit gekommen syen, wenn nicht seine uns nähere Lage, die Trümmer seiner Alterthümer, vorzüglich aber die Sagen der Griechen es dahin gebracht hätten. $[\ldots]^{\text {ç37 }}$

Herder hatte den Ägyptern ihre Stelle in der Reihe der Völker angewiesen. Sein universalhistorisches Gebäude nahm sie weit heraus aus dem Gesichtskreis der Antike. Mehr noch: sie wurden entzaubert, mit nüchternem Verstande aus der Verbindung des klassischen Altertums ausgestoßen. Diese Lehre war folgenschwer. Sie hat zwar nicht gekonnt, Winckelmanns Bild der Antike völlig auszulöschen. Die Kunstbetrachtung knüpfte an dieses Bild auch später noch an. Aber die historische Forschung konnte Ägypten nicht länger als Glied der Antike empfinden. Wie die Ägypter, so spaltete sie auch die Etrurier vom klassischen Altertum ab. Und während dieses mit Griechen und Römern nach wie vor Inhalt und Macht der geistigen Bildung blieb, wurden Ägypter und Etrurier völkergeschichte Objekte. Und ihre Hinterlassenschaften sanken zu Kuriositäten herab. ${ }^{38}$

Herder hat in hervorragender Weise das maßstabgerechte begreifen geschichtlicher Phänomene als solcher ermöglichen helfen. Damit soll gesagt sein, was für das künftige Verhältnis zum alten Ägypten von der größten Bedeutung ist: dass er den Bereich der antiken Vierheit gesprengt und zugleich den Bezirk der Kunst dem Lebensganzen untergeordnet und jede gesellschaftliche Einheit und Ganzheit aus ihr selbst heraus, aus der Summe der Bedingungen ihrer Existenz zu verstehen gefordert hat. Hier

\footnotetext{
${ }^{37}$ Karl Heinrich Ditunanu. Goethe und die „Egyptischen Sachen“. In: Mittelungen des deutschen Instituts für Ägyptische Altertumskunde. Kairo 1943, S. 96-106. Hicr: S. 104.

${ }^{38}$ Ebd. S. $104 f$.
} 
liegt das Entscheidende. Und es findet in der Nutzanwendung auf Ägypten bezeichnenderweise bereits in Winckelmanns Werk. ${ }^{39}$

Dabei hat Herder selber eine Wandlung erlebt. In seiner Schrift von 1768 über Winckelmann wirft er diesem noch vor, dass er die Ägypter überall mit griechischen Augen sieht und am Ideal der Griechen misst: „So sehr Grieche ist selbst Herodot kaum". Herder sieht in Hermes Trismegistos den symbolischen Erfinder der Zahlen und Buchstaben und veröffentlicht noch 1801 in seiner Zeitschrift „Adrestea“ einen ,hermetischen“ Dialog zwischen Hermes und Pymander. ${ }^{40}$

Herder spricht von „Ruhe“ und „Tod“, die in den Statuen herrschen. Und vom Abscheu der Ägypter gegen transitorische Momente in den Bildwerken. Damit trifft er exakt das, was man heute als "Zeitferne" der ägyptischen Kunst hervorhebt. An einer anderen Stelle, kurz vorher, erwägt er mit gesundem Urteil Zusammenhänge zwischen dem alten ägyptischen und griechischen Stil, was man heute auf der Weise noch zu tun haben und tun. Herder in seinem starken historischen Sinn der Quellenlage seiner Zeit im Hinblick auf Ägypten bewusst gewesen. Er will sich, um den Anfang der Mythologie bemüht, nicht weiter damit beschäftigen. Was er mit seinen „vorägyptologischen“ Mitteln, zu dem bei dem umfassenden Konzept seines großen Wurfes doch nur im Vorübergehen, über Ägypten wohl gemerkt: die handwerkliche und religiöse Bindung der Kunst, das Naturjahr als Grundlage des Kalenders, die Formation der Priester als eines Standes „Ordens", den konservativen Zug : zur Institutionalisierung des Alten. Daraus die relativ bereit geschilderte Hemmung der Wissenschaft. Beim letzten Punkte muss freilich eingehalten. Und Herder gegen Verdacht in Schutz genommen werden. Er hat Ägyptens Leistung abgewertet. Gewiss finden es sich harte Worte. Sie zielen auf die in den vergangenen Jahrhunderten fast angebeteten Hieroglyphen:

Hieroglyphen sind der erste rohe Kindesversuch des menschlichen Verstandes, der Zeichen sucht, um seine Gedanken zu erklären [...], daß aber die Ägypter so lange bei dieser unvollkommenen Schrift blieben und sie Jahrhunderte hin [...] auf Felsen und Wände malten, welche Armut von Ideen, welch ein Stillstand des Verstandes zeigt dieses! Wie enge musste der Kreis von Kenntnissen einer Nation und ilbres weitläufigen gelehrten Ordens sein. [...] Dürfen wir uns also wundern, daß ein so schriftarmes and doch nicht ungeschicktes Volk sich in mechanischen Künsten hervortat? Der Weg zur wissenschaftlichen Literatur war ihnen

\footnotetext{
${ }^{39}$ Morenz, S. $132 f$.

${ }^{40}$ Erik Hornung. Esoterische Ägypten. München 1999, S. 134.
} 
durch die Hieroglyphen versperret, und so musste sich ihre Aufinerksamkeit desto mehr auf sinnliche Dinge richten. ${ }^{\text {(4l }}$

Hier in seinen „Ideen zu einer Philosophie der Geschichte der Menschheit" (1784-1791) verfällt Herder in den gleichen Fehler wie Winkelmann. Dabei hat er in der Wertung von Morenz ${ }^{42}$ dem "maßstabgerechten Begreifen geschichtlicher Phänomen" Bahn gebrochen und manche durchaus richtige Einsicht in das Wesen der altägyptischen Kultur formuliert. Er sieht aber in Ägypten und seinen „Hieroglyphen nur einen ersten rohen Kinderversuch des menschlichen Verstandes", denn auch „die rohesten Wilden in Amerika hatten Hieroglyphen", wobei er an Mexikaner und ihre Bilderschriften denkt. Heute wird wohl niemand mehr die Azteken oder Maya „roheste Wilde“ bezeichnen.

In einem Brief von 1826 findet auch Goethe, dass Mumien nicht gerade ein „unerlässliche:: Bestandteil eines Museums" seien, sondern ein „Modeartikel, und die Mode spricht: Was viele haben, muß jeder haben. $\mathrm{Zu}$ was es nutzt, fragt niemand“. In seinen späten Jahren sind ihm ägyptische, wie auch indische und chinesische Altertümer, ,,immer nur Curiostäten; es ist sehr wohl gethan, sich und die Welt damit bekannt zu machen; zu sittlicher und ästhetischer Bildung aber werden sie uns wenig fruchten. ${ }^{\text {c4 }} 3$

Dann folgen Sätze über die Lehren, die die Ägypter durch die Natur selbst empfingen und auch zu Naturjahr und Kalender führten. Herder fährt in seinem Werk fort: „Was bei den Ägyptern die Hieroglyphen dazu konnten, war-der Wissenschaft eher schädlich als nützlich" ". Damit ist er beim Achtergewicht seines Anliegens und schlägt abschließend die Hieroglyphen - Weisheit im allgemeinen und die neurer Zeiten im besonderen kräftig zusammen. Gerade daraus aber erklärt sich ganz natürlich die Härte der Kritik. Sie richtet sich, höchst aktuell, gegen die Schwarmgeisterei um ägyptische Weisheit, die man vorrangig in den Hieroglyphen suchte, eben weil sie nicht lesen konnte. So sagt Herder, wie ähnlich lange vor ihm Leibniz über die Obeliskeninschriften rundheraus: „Was würde, was könnte man an ihnen anders, als etwa eine Chronik verstorbener Begebenheiten oder eine vergötternde Lobschrift ihrer Erbawer? [...] “" Von der Aktualität dieses Schwärmens wie seiner ins Bedenkliche reichenden Wirkensbreite vom edlen Streben zu menschlicher Erziehung.

Die Vertiefung des Lebens bis zur blanken Gaunerei zeugen die „Mysterien der Egyptier“ des Wiener Freimaurerhaupts I. von Born, der

11 Zit. nach Morenz, S. 134.

42 In seinem Buch „Begegnung Europas mit Ägypten“, Stuttgart 1999, S. 134ff.

${ }^{43}$ Erik Homung. Das esolerische Ägyplen. München 1999, S $135 f$. 
mit Ethos und Weihe der Zauberflöte inspiriert hat. Aber auch der "Großenkophta" Giuseppe Balsamo, der und Anderen in Basel eine "ägyptische Loge" gegründet hat. Was darüber hinaus, noch handgreiflicher, an Richtungskämpfen zwischen Klarheit und Qualm in der zeitgenössischen Freimaurerei, der Herder wie Goethe verbunden war. Es erklärt sich der äußere Anlass der Kritik und auch deren Breite aus der Kampfsituation Herders. So hat sie ihren eigentlichen Grund auf dem festesten Felsen, den die Wahrheit für Denker und Forscher geben kann. ${ }^{44}$

Herders Ägyptens Bild ist also weder schwärmend noch abwertend, sondern für seine Informationsquellen erstaunlich. Wahrscheinlich hatte er gerade für Ägypten den rechten Griff, weil es gegenüber einer mächtigen Tradition in das Recht einzusetzen. Andererseits aus dem verkiärenden Nebel angeblicher Weisheit in eine zunächst bescheidene Wirklichkeit zu rücken war. Man soll hier Herder einen Ehrenplatz geben, der die geistigen Positionen gesetzt hat, von denen aus die Geistwissenschaften über Nilland, Ägyptologie und Archäologie aufgebaut werden konnte. ${ }^{45}$

Auf Goethe hatte Herders Lehre, wie man es weiß, maßgeblichen Einfluss. Wenn man heute bemerken, dass Goethe sich nach Beendigung der Italienischen Reise von ägyptischen Dingen abwandte, so ist die Urasche dazu in Herders weltgeschichtlicher Ordnung zu suchen. Zwar zielen Goethes geringschätzige Bemerkungen im wesentlichen auf Erscheinungen der ägyptischen Religion und Hieroglyphe, so dass Viele Experten $^{46}$ meinten, dass es der ägyptischen Kunst gerechter geworden wäre. Aber das Problem liegt ja, wie man sah. Es ist mehr tiefer. Die ägyptische Kunst erlebte und bewunderte Goethe in Rom als Teil des ihm von Winckelmann überkommenen antiken Weltbildes. Als dieses Bild durch Herder zerstört war, wurde von Goethes Abneigung nicht nur ägyptische Religion und Hieroglyphe, sondern auch die ägyptische Kunst betroffen. So dass er in der Anzeige der Propyläen 1799, also zwölf Jahre nach seiner Rückkehr aus Italien, die Meinung verrat: Fachleute Meinung nach halten sich Liebhaber gewöhnlich viel zu lange bei den ägyptischen, ältest griechischen, alt italienischen, besonders aber der altdeutschen Kunst auf, deren Verdienste meist nur ein historisches, selten ein höheres

\footnotetext{
${ }^{44}$ Denn wir wissen seit längerer Zeit, dass ägyptische Wissenschaft einerseits mit frühen giten Würfen eingesetzt hat, aber dann eher stecken blieb, dass die in Hieroglyphen gewahrte Tradition also den Fortschritt hinderte. Auch haben wir gesehen, wie anderseits ihre bedeutenden Leistungen praktischer und nicht denkerisch-mathematischer Natur waren. So lassen sich denn zeitgebundener Anlass und dauerhaft-sachlicher Grund für Herders Kritik mühelos ermitteln. Dabei zeigen sich, dass der Kritiker in wichtigen Dingen recht hat.

${ }^{45} \mathrm{Vgl}$. Morenz, S. $135 f$.

${ }^{46}$ Max Piepers Aufsatz „Goethes Brief über Ägypten“. In: den Mitteilungen des Institutes für ägyptische Altertümer Kairo 1932. Und Volkmanns Aufsatz „Goethe und Ägypten“. In: Zeitschrift für Ägyptisclie Sprache, Berlin und Leipzig 1936.
} 
Kunstinteresse haben. Goethe, der in Rom mit so viel Eifer immer wieder „in die Egyptischen Sachen“ gekommen war, schrieb bereits 1797 in einem Brief an Dr. Schleusner:

" [...] Mit den ägyptischen Altertümer mache man sich aus Reisebeschreibungen mit so wenig Kosten als möglich bekannt. [...] $]^{\text {‘47 }}$

Wenn er 1808 in den „Unterhaltung über verschiedene Gegenstände der Kunst" der Auffassung ist:

" [...] daß in den ältesten Zeiten die Kunst jederzeit der Religion diente, in dem sie gewisse strenge, trübe, seltsame und gewaltsame Vorstellungen ausbildete, daß deswegen die bildende Kunst nirgends vom Natürlichen anfing, sondern überall mit einer Art von barbarischem Sinn und Geschmack“, und dass „die Ägypter sich auch aus der Knechtschaft dieses dunkeln Zustandes nie befreiten, $[\ldots]^{\text {‘ } 48}$

So sind solche Sätze ganz unter dem Eindrack der "Herders Ideen“ geschrieben, wie denn auch im dritten Buch von „Wilhelm Meisters Wanderjahren" die Bezeichnung der ägyptischen, indischen und chinesischen Altertümer als Kuriositäten Herders Einfluss erkennen lässt. Nur einmal noch wird der ägyptischen Kunst ein zumindest betrachtendes Interesse gewidmet, und zwar in dem Aufsatz über Winckelmann, vor dessen Meisterschaft offenbar noch einmal das in Rom erlebte Bild der antiken Welt in Goethes Seele aufbrach. ${ }^{49}$

Wie es oben gesagt ist, hätte Herder sich in einem Essay lediglich gegen den Stil der ägyptischen Kunst ausgesprochen, so würde das vermutlich an Goethes eigenem Erlebnis ägyptischer Kunst abgeprallt sein. Die Durchsprechung des bisherigen Bildes der antiken Welt jedoch bewirkte bei Goethe die Aufgabe seines in Rom erworbenen Verhältnisses zur ägyptischen Kunst. Und dies geschah um so eher, als das Jahrzehnt nach Italien für Goethe olnehin spannungsreich war und ihn noch manches andere in düstere Zweifel ziehen ließ. Man vergisst nicht, dass in jenen Jahren "Die Braut von Korinth" und „Der Gott und Bajadere“ entstanden. ${ }^{50}$

Dennoch sind seiner Phantasie ägyptische Kunstformen immer gegenwärtig geblieben. So benutzt er in der "Geschichte der Farbenlehre" (1810 beendet) im Abschnitt „Überliefertes" die Formen der Pyramide und des Obelisken zu Gleichnisbildern für Platos und Aristoteles

\footnotetext{
${ }^{47}$ Volkmann, S. 7.

${ }^{48}$ Dieser Aufsatz über Winckelmann war im Jahre 1805.

${ }^{49}$ Dieser Aufsatz über Winckelmann war im Jahre 1805.

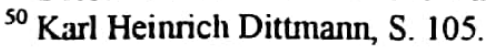


Verhalten zur Welt: „(Aristoteles) umzieht einen ungeheueren Grundkreis für sein Gebäude, schafft Materialien von allen Seiten her, ordnet sie, schicht sie auf und steigt so in regelmäßiger Form pyramidenartig in die Höhe, wenn Plato, einen Obelisken, ja einer spitzen Flamme gleich, den Himmel sucht. “51

Das durch Winckelmann fixierte Bild der antiken Welt, das Ägypten so selbstverständlich einschloss, und die von Herder entworfene weltgeschichtliche Ordnung, die Ägypten aus der ideellen Verknüpfung entrückte, bestimmten fortab als Antinomie das geistige Verhältnis zum ägyptischen Altertum und ließen es in jenem Schwebezustand, der im Grunde selbst heute noch das geistige Empfinden des Abendland gegenüber dem alten Ägypten bezeichnet. Denn während in der Kunstbetrachtung und in den Kunstschöpfung der Folgezeit, besonders in der Architektur, bei aller Bevorzugung der griechischen Welt der vierfache Begriffsinnhalt der Antike noch lange Zeit nachwirkte, verlor der philologische und historische Gesichtskreis die Füllung mit dem ägyptischen Altertim.

Champollions geniale Entzifferung der Hieroglyphen, die mit einem Scllage die Archive Ägyptens öffnete, fand infolgedessen zunächst ein erstaunlich wenig aufnahmefreudiges Publikum. Ägypten war im wissenschaftlichen Denken verblasst. Die verhängnisvolle Entrückung Ägyptens machte sich damals bereits in der wissenschaftlichen Behandlung der ägyptischen Kunst bemerkbar. Die Künste Ägyptens, Persiens, Indiens werden durchaus in Herders Sinne als äquivalente völkergeschichtliche Erscheinungen angefübrt, von einer Kennzeichnung Ägyptens als Glied der nahe angelvenden klassischen Antike ist nicht mehr die Rede.

Das jenige Buch, das Ägypten wieder herbeiholt und ihm seine überragende geschichtliche Bedeutung zurück gibt, den an Herder anklingenden Titel trägt: „Ägyptens Stelle in der Weltgeschichte“ von Carl Josias von Bunsen (1845). Dieses Buch ist unter Mitwirkung von Richard Lepsius zustande gekommen. Aber wenn auch durch Bunsen und Lepsius die wissenschaftliche Ägyptenkunde, in historischem, philologischem, religions- und kunstgeschichtlichem Sinne, wieder den erhöhten Platz in Geschichtsbilde erhielt. Sie blieb doch schließlich reine Wissenschaft, während für Winkelmann die Kunst der Ägypter ein Teil seines Humanismus war. Und während in Übereinstimmung mit seiner Konzeption ägyptische Formen, wie Obelisken, Sphinx und Pyramiden, Elemente des spätbarocken und klassizistischen Kunstschaffens wurden und damit $\mathrm{zu}$ dem am Altertum orientierten Lebensgefühl jener Zeit gehörten, konnte das große Ereignis der Lepsius-Expedition nach

\footnotetext{
${ }^{51}$ Zit. nach Karl Heinrich Dittmann. Goethe und die „Egyptischen Sachen“. In: Mittelungen des
} deutschen Instituts für Ägyptische Altertumskunde. Kairo 1943, S. 96-106. Hier: S. 105. 
Ägypten (1842 - 1842) zwar die Wissenschaft entscheidend zu fördern, aber nicht die zeitgenössische Kunst und Kultur zu befruchten. Die Nachbildung eines ägyptischen Tempels wurde bezeichnenderweise in Berlin ins Museum eingebaut. ${ }^{52}$

Das Schicksal eines Gedankenguts, an dem Goethe teilhatte, bis zu dem Punkte zu verfolgen, von dem aus es sich in menschlichen Denkens eingliedert. Zum anderen weist das Denken Goethes, das in dem behandelten Thema zwischen Winckelmann und Herder steht, selbst weit über sich hinaus. Weil der Strahl der ägyptischen Kunst, den das Prisma Goethes Geist empfangen hat. Nicht wie andere Strahlen haben farbenreich und vielfältig gebrochen, sondern abgelenkt wurde, drängte es uns, nach den Gründen zu fragen. Sie liegen außerhalb Goethes, im Geschehen des deutschen Geisteslebens. Dieses Geistesleben hatte nach Goethes Zeit damals seiner breiten Kleidung aufgetrennt, um es anders neu zu wirken. ${ }^{53}$

Herder war als junger Mensch anderer Ansicht gewesen. In seinen Jugendschriften batte er noch nach wunderbaren Offenbarungen gesucht, die sich in den Schriften der alten Ägypter zeigen sollten. Der reife Mann war kritischer geworden. Er sucht nicht nach Dingen, die der Natur der Sache nach hier nicht zu finden waren.

Bei der engen Verbindung, in der Goethe mit Herder gestanden hat, ist das an und für sich wahrscheinlich. Dass er die Grundanschauungen der Ideen geteilt hat. D.h. hier von der Kindlichkeit der betreffenden Völker ist wohl sicher die begeisterten Worte, mit denen er Herders Werk aufgenommen, zeugen dafür. Von Herders Standpunkt aus konnten die Ägypter zunächst keine besondere Anerkennung finden. Sie gehörten in die Kindheit des Menschengeschlechtes als eine Auffassung, die wie man weiß nur sehr bedingt richtig ist. So hatten sie der Gegenwart nichts zu sagen. So glaubte man einigermaßen klargestellt, wie wenig die Zeit geeignet war, sich tiefer in die Geschichte und in das Wesen des ägyptischen Volkes zu versenken. Goethe fand zunächst einen wüsten Haufen von allerhand Nachrichten über das alte Ägypten ohne jeden Zusammenhang vor. Und später als durch Herder eine historische Auffassung angebaut war, war das Ergebnis zunächst nicht dazu angetan. Es ist für ihn zu tieferem Eindringen zu ermutigen. Die Entzifferungsarbeiten Champollions kamen für Goethe in Weimar zu
spät. $^{5}$

\footnotetext{
52 Vgl. Karl Heinrich Dittmann. Goethe und die „Egyptischen Sachen“. In: Mittelungen des deutsclicn

Instituts für Ägyptische Altertumskunde. Kairo 1943, S. 96-106. Hier: S. 106.
${ }^{54}$ Ebd. S. 106.

${ }^{54}$ Vgl. Max Pieper. Goethes Unteilen über ägyptische Kunst und Religion. In: Mitteilungen des

Deutschen Instituts für Ägyptische Altertumskunde II., Kairo 1932, S. 117- 134. Hier: S. 132.
} 
Ibrahim Hamed Abd Ella

Dozent an der Abteilung fúr Germanistik

Al Alsun Fakultăı

Al Minia Universität

Internationale Tagung der Al-Alsun Fakultät

Al-Minia Universităt

Das Ägyptenbild in der Weltliteratur

14 - 15 April 2004

\section{Zusammenfassung}

\section{Zum Bild der altägyptischen Kultur im Leben und Werk des großen deutschen Dichters \\ Goethe}

In Goethes Gesamtwerk spielt die altägyptische Kultur eine zentrale Rolle. Man hat öfters schmerzlich empfunden, dass die Rezeption der altägyptischen Kultur nicht nachhaltig von Goethes Geist aufgenommen und reflektiert worden ist. Goethe legte großes Gewicht auf die Kultur der Antike. Man meint, Goethe wäre der altägyptischen Kultur gerecht gewesen, wenn er dazu die Möglichkeit erlebt hätte.

Diese Untersuchung befasst sich mit Goethes Verhältnis zur altägyptischen Kultur. Der deutsche Maler Wilhelm Tischbein, der französische Maler Cassas und andern öffneten Goethes Augen auf die alte Kunst Ägyptens. Die Anfänge Goethes Begegnung mit der altägyptischen Kultur erfuhr er von seinen Reisen in Italien. Sein Ägyptenbild erhielt er ferner von seiner eigenen lektüre, vor allem, der Lektüre des Winkelmannes Werk "Geschichte der Kunst des Altertums" sowie des Werkes seines Lehrers Johann Gottfried Herder: „Ideen zur Geschichte der Menschheit". Die beiden Dichter übten auf ihn einen großen Einfluss aus. 


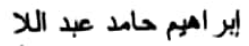

$$
\begin{aligned}
& \text { مدرس بقعم اللغة الالمانبة } \\
& \text { كلية الالمن - جامعة المنبا }
\end{aligned}
$$

\section{ملخص}

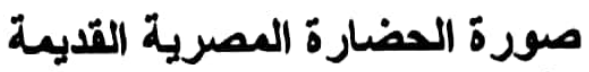 \\ لفى حياة جوته، شاءر الماتيا الأكبر \\ و نتاجه الألبى}

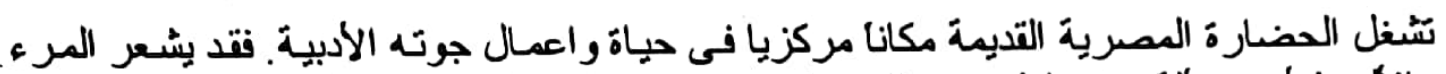

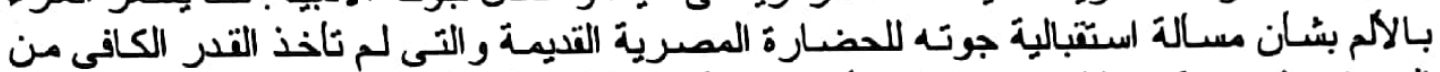

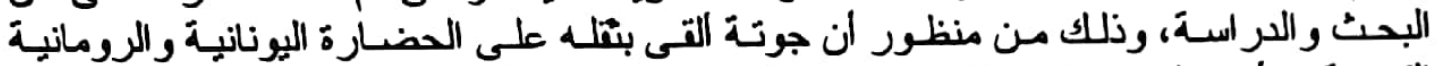

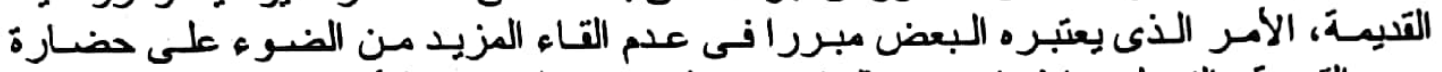

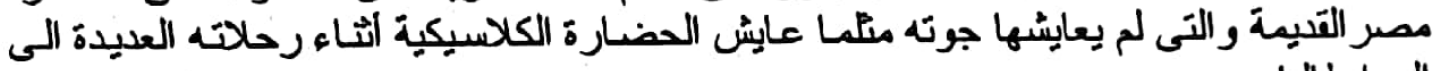
الى إيطاليا.

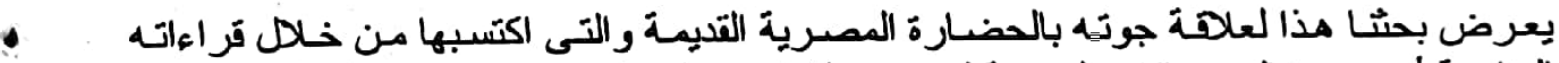

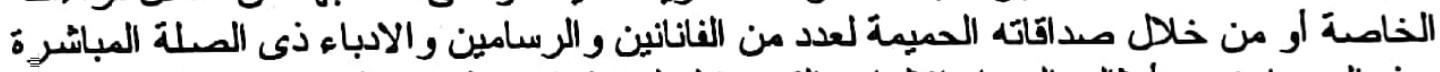

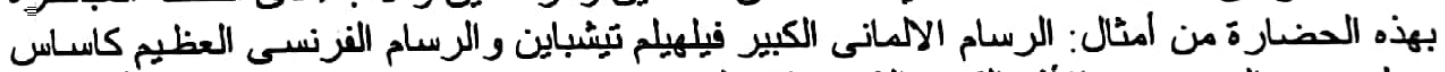

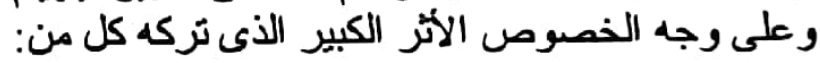

( ) الأديب و الناقد و المور خ فينكلمان و كتابه: تاريخ فن العصور القديمة.

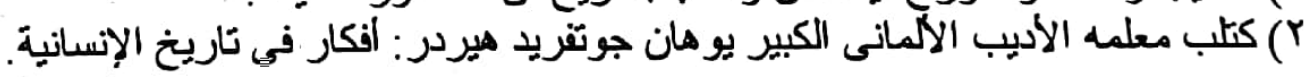

